\title{
FIXED POINT THEOREM FOR GENERALIZED SET-VALUED CONTRACTIONS ON PARTIAL METRIC SPACES
}

\author{
MOSTEFA DJEDIDI, ABDELOUAHAB MANSOUR AND KHADRA NACHI
}

\begin{abstract}
In this paper, a new version of Ekeland's variational principle on partial metric spaces is given. Using this variational principle, we establish a general result on the existence of a fixed point for a class of genralized set-valued contractions on partial metric spaces, other results are also given.
\end{abstract}

\section{Introduction and preliminaries}

Over the last decades, fixed point theory has shown to be a very powerful tool in the study of nonlinear phenomena. In particular, fixed point techniques have been developed in pure and applied analysis, topology and geometry. Among the most famous results of this theory, the Banach contraction principle [11] has been extensively studied and generalized in several settings. The variety of concepts and methods involved corresponds to the multiplicity of purposes. Recently, studies on the existence and uniqueness of fixed points of self-mappings on partial metric spaces have gained momentum ([36], [49], [26]). Partial metric space have been introduced by Mathews [28] in 1992 as a part of the study of denotational semantics of dataflow networks (see $[29,30]$ ). In fact, partial metric spaces play an important role in constructing models in the theory of computation and also to model metric spaces via domain theory (see, [14], [15], [25], [29]).

In 1994, Mathews [29] showed that the Banach contraction principle can be generalized to partial metric spaces in view of applications in program verification. This remarkable paper of Mathews constructed another important bridge between the domain theory in computer sciences and fixed point theory in mathematics (see [40]). Later on, many researchers studied fixed point theorems in partial metric spaces, particularly Oltra-Valero [36, 2004], Rus [39, 2008], Ćirić et al. [15, 2011], Romaguera [38, 2012], Aydi et al. [4, 2012].

Received Januery 27, 2017, accepted June 9, 2017.

2010 Mathematics Subject Classification. Primary 47H09 54E50, Secondary 37C25, 47H10.

Key words and phrases. Partial metric space, fixed point, generalized set valued, Ekeland's variational principle.

Corresponding author: Abdelouahab Mansour. 
Our purpose in this paper is to give a new result on the existence of a fixed point for a class of set-valued mappings satisfying a generalized contraction condition on a partial metric space. Here our method relies on the Ekeland variational principle [17, 1972] (see also, $[18,19])$ and not on the classical method Banach-Picard. With this aim, we first show that the classical Ekeland's variational principle can be generalized to partial metric spaces. Ekeland's variational principle (in short, EVP) is one of the most applicable results of nonlinear analysis: it is used for problems from fixed point theory, optimization, optimal control theory, game theory, nonlinear equations, dynamic systems, etc; see, for example, ([1]-[2], [12], [13], [17][20], [23], [27], [33], [35], [47]) and the references therein. Recall that the classical statement of Ekeland's variational principle is as follows:

Theorem 1 ([11], [8] Theorem 2.1, [35] Theorem B). Let $(X, d)$ be a metric space. Then the following properties are equivalent:

(1) $(X, d)$ is complete,

(2) Every proper, lower semicontinuous and bounded from below function $f: X \rightarrow \mathbb{R} \cup\{\infty\}$ admits a $d$-point that is, there exists $x \in X$ such that

$$
f(x)<f(y)+d(x, y) \quad \forall y \in X, y \neq x .
$$

Let us start by recalling some basic definitions and properties of partial metric spaces which can be found in $[29,28]$.

Definition 2. A partial metric on a nonempty set $X$ is a function $p: X \times X \rightarrow \mathbb{R}_{+}$such that for all $x, y, z \in X$ :

(i) $x=y \Longleftrightarrow p(x, x)=p(x, y)=p(y, y)$,

(ii) $p(x, x) \leq p(x, y)$,

(iii) $p(x, y)=p(y, x)$,

(iv) $p(x, z) \leq p(x, y)+p(y, z)-p(y, y)$ (triangle inequality).

A partial metric space is a pair $(X, p)$ such that $X$ is a nonempty set and $p$ is a partial metric on $X$ (called a $p$-metric).

From the definition of a partial metric, $p(x, x)$ is not necessarly zero so that $p$ is not a metric. But it is clear (from (ii) and (i)), that if $p(x, y)=0$ then $x=y$.

A simple example of a partial metric space is the pair $\left(\mathbb{R}_{+}, p\right)$, where $p(x, y):=\max (x, y)$ for all $x, y \in[0, \infty)$. Another example is the pair $(X, p)$ with $X:=\{[a, b] \subset \mathbb{R}: a \leq b\}$ and $p([a, b],[c, d]):=\min (b, d)-\min (a, c)$. 
Notice that for a partial metric $p$ on $X$, the function $d_{p}: X \times X \rightarrow[0, \infty)$ given by

$$
d_{p}(x, y):=2 p(x, y)-p(x, x)-p(y, y)
$$

is a (usual) metric on $X$. Moreover, $c_{p}: X \rightarrow \mathbb{R}_{+}$given by $c_{p}(x):=p(x, x)$ is a nonexpansive function in the sense that for any $(x, y) \in X^{2}$ one has $|c(x)-c(y)| \leq d_{p}(x, y)$. One has a converse:

Proposition 3. Given a nonexpansive function c on a metric space $(X, d)$ a partial metric on $X$ is obtained by setting for $x, y \in X$

$$
p_{d, c}(x, y):=\frac{1}{2}[d(x, y)+c(x)+c(y)] .
$$

Moreover, the map $(d, c) \mapsto p_{d, c}$ is a bijection between the set of pairs $(d, c)$ as above and the set of partial metrics whose inverse is the map $p \mapsto\left(d_{c}, c_{p}\right)$.

Observe that each partial metric $p$ on $X$ generates a $T_{0}$ topology $\tau_{p}$ on $X$ (see [29, p. 187]) which has as a base the family of open $p$-balls $\left\{B_{p}(x, \varepsilon), x \in X, \varepsilon>0\right\}$ where $B_{p}(x, \varepsilon):=$ $\{y \in X, p(x, y)<p(x, x)+\varepsilon\}$ for all $x \in X$ and $\varepsilon>0$. Similarly, the closed $p$-ball is defined by $B_{p}[x, \varepsilon]:=\{y \in X, p(x, y) \leq p(x, x)+\varepsilon\}$.

Definition 4. Given $(X, p)$ a partial metric space and $\left(x_{n}\right)$ a sequence in $X$. We will say that:

(a) $\left(x_{n}\right)$ converges to $x \in X$ and we note $x_{n} \stackrel{p}{\rightarrow} x$ if $\lim _{n \rightarrow \infty} p\left(x_{n}, x\right)=p(x, x)$.

(b) $\left(x_{n}\right)$ is a Cauchy sequence if $\lim _{n, m \rightarrow \infty} p\left(x_{n}, x_{m}\right)$ exists and is finite.

(c) The partial metric space $(X, p)$ is complete if every Cauchy sequence $\left(x_{n}\right)$ in $X$ converges, with respect to $\tau_{p}$, to a point $x \in X$ such that $p(x, x)=\lim _{n, m \rightarrow \infty} p\left(x_{n}, x_{m}\right)$.

The following lemma is useful, it highlights the link between the definition above and the standard one in metric spaces.

Lemma 5 ([29]). Let $(X, p)$ be a partial metric space and let $d_{p}$ be the metric given by (2). Then

(1) $\left(x_{n}\right)$ is a Cauchy sequence in $(X, p)$ if and only if $\left(x_{n}\right)$ is a Cauchy sequence in $\left(X, d_{p}\right)$.

(2) A partial metric space $(X, p)$ is complete if and only if the metric space $\left(X, d_{p}\right)$ is complete. Furthermore, $\lim _{n \rightarrow \infty} d_{p}\left(x, x_{n}\right)=0$ if and only if

$$
p(x, x)=\lim _{n \rightarrow \infty} p\left(x, x_{n}\right)=\lim _{n, m_{\rightarrow} \infty} p\left(x_{n}, x_{m}\right) .
$$




\section{Ekeland's variational principle on partial metric space}

In this section, we establish an Ekeland's variational principle on partial metric spaces. From this general EVP, we give in next section, a general fixed point theorem for set-valued mappings satisfying some contraction condition. Let us first introduce the following definition:

Definition 6. Given $(X, p)$ a partial metric space and $f: X \rightarrow \mathbb{R} \cup\{+\infty\}$ a function. A point $x \in \operatorname{dom} f:=f^{-1}(\mathbb{R})$ is said a $p$-point of $f$ if

$$
f(x)+p(x, x)<f(y)+p(x, y) \quad \forall y \in X, y \neq x / p(y, y)=p(x, x) .
$$

Note that if $p:=d$ is a metric, this definition coincides with the usual one (1). Observe that $p$-points are in $\operatorname{dom} f$, and that global minima of $f$ are $p$-points. Indeed, consider $x$ is a global minima of $f$ and $y \neq x$ such that $p(y, y)=p(x, x)$. Hence, $p(x, y)>p(x, x)$ and we obtain

$$
f(x) \leq f(y)<f(y)+p(x, y)-p(x, x)
$$

that is $x$ is a $p$-point of $f$. Observe too that if $x$ is a $d_{p}$-point of $f$, it is a $p$-point of $f / 2$.

Let $f: X \rightarrow \mathbb{R} \cup\{+\infty\}$ be a function defined on a partial metric space $(X, p)$. We will say that $f$ is lower semicontinuous (l.s.c.) on $X$ if for all $x$ and any sequence $\left(x_{n}\right)$ in $X$, one has

$$
p\left(x_{n}, x\right) \rightarrow p(x, x) \Longrightarrow f(x) \leq \liminf _{n \rightarrow \infty} f\left(x_{n}\right) .
$$

Consider now the set

$$
S(x):=\{y \in X / p(y, y)=p(x, x) \text { and } f(y)+p(y, x) \leq f(x)+p(x, x)\}
$$

which is nonempty $(x \in S(x))$ and let $\hat{f}:=f_{\mid S(x)}$ the restriction of $f$ to $S(x)$.

Proposition 7. We have the following properties:

(1) $x$ is a $p$-point of $f$ if and only if $S(x)=\{x\}$,

(2) If $\hat{x}$ is a p-point of the restriction $\hat{f}$ on $S(x)$ then $\hat{x}$ is a p-point of $f$ on $X$,

(3) If $y \in S(x)$ then $S(y) \subset S(x)$,

(4) If $f$ is lower semicontinuous on $(X, p)$ then for any $x, S(x)$ is closed in $\left(X, d_{p}\right)$ that is

$$
\forall\left(x_{n}\right) \subset S(x), \quad \lim _{n \rightarrow \infty} d_{p}\left(x_{n}, \bar{x}\right)=0 \Longrightarrow \bar{x} \in S(x) .
$$

\section{Proof.}

(1) It is clear that if $S(x)=\{x\}, x$ is a $p$-point of $f$. Conversely, consider a point $y \in X$ such that $y \neq x$. If $p(y, y) \neq p(x, x), y \notin S(x)$. Assume that $p(y, y)=p(x, x)$ so $f(x)+p(x, x)<$ $f(y)+p(y, x)$ (by the assumption) i.e., $y \notin S(x)$. 
(2) Given $\hat{x}$ is a $p$-point of $\hat{f}$ on $S(x)$ and $y \in X, y \neq \hat{x}$ such that $p(y, y)=p(\hat{x}, \hat{x})$. Hence, $p(y, y)=p(x, x)$. If $y \in S(x)$ then (4) is satisfied for $\hat{x}$. Suppose now $y \notin S(x)$ and as $\hat{x} \in S(x)$, we get the conclusion since

$$
f(\hat{x})+p(x, \hat{x}) \leq f(x)+p(x, x)<f(y)+p(y, x)
$$

(3) Let $y \in S(x)$ and $z \in S(y)$ so $p(z, z)=p(y, y)=p(x, x)$ and

$$
\begin{aligned}
f(z)+p(x, z) & \leq f(z)+p(x, y)+p(y, z)-p(y, y) \\
& \leq f(y)+p(y, x) \leq f(x)+p(x, x)
\end{aligned}
$$

that is $z \in S(x)$.

(4) Assume $f$ is lower semicontinuous and fix $x$ in $X$. Consider a sequence $\left(x_{n}\right)$ in $S(x)$ such that $\lim _{n \rightarrow \infty} d_{p}\left(x_{n}, \bar{x}\right)=0$. Thus $p(\bar{x}, \bar{x})=\lim _{n \rightarrow \infty} p\left(\bar{x}, x_{n}\right)=\lim _{n \rightarrow \infty} p\left(x_{n}, x_{n}\right)=p(x, x)$ since $x_{n} \in S(x)$ for all $n$. Moreover, using the following triangular inequality,

$$
p(\bar{x}, x) \leq p\left(\bar{x}, x_{n}\right)+p\left(x_{n}, x\right)-p(x, x)
$$

we get

$$
p(\bar{x}, x) \leq \liminf _{n \rightarrow \infty} p\left(x_{n}, x\right)
$$

In addition, by (6), we have

$$
f\left(x_{n}\right)+p\left(x_{n}, x\right) \leq f(x)+p(x, x)
$$

and the lower semicontinuity of $f$ leads to

$$
f(\bar{x})+p(\bar{x}, x) \leq \liminf _{n \rightarrow \infty}\left(f\left(x_{n}\right)+p\left(x_{n}, x\right)\right) \leq f(x)+p(x, x)
$$

that is $S(x)$ is closed.

We give now our main result about Ekeland's variational principle on partial metric spaces.

Theorem 8. Let $(X, p)$ be a complete partial metric space and let $f: X \rightarrow \mathbb{R} \cup\{\infty\}$ be a proper, l.s.c. and bounded from below function. Let $x_{0} \in \operatorname{dom} f$ and $\lambda>0$ be fixed. Then there exists $\bar{x} \in X$ such that $p(\bar{x}, \bar{x})=p\left(x_{0}, x_{0}\right)$ and

(1) $f(\bar{x})-f\left(x_{0}\right) \leq \lambda\left(p\left(x_{0}, x_{0}\right)-p\left(\bar{x}, x_{0}\right)\right) \leq 0$,

(2) $f(\bar{x})+\lambda p(\bar{x}, \bar{x})<f(y)+\lambda p(\bar{x}, y) \quad$ for all $y \in X, y \neq \bar{x}$ such that $p(y, y)=p(\bar{x}, \bar{x})$ that is $f$ admits a p-point. 
Proof. Without loss of generality we may suppose $\lambda:=1$. For $x \in \operatorname{dom} f \operatorname{define} v(x):=\inf _{y \in S(x)}$ $f(y)>-\infty$ since $f$ is proper and bounded from below. Let $x_{0} \in \operatorname{dom} f$ and assume that $x_{0}$ isn't a $p$-point (otherwise there is nothing to prove). There exists then $x_{1} \in S\left(x_{0}\right), x_{1} \neq x_{0}$ such that $f\left(x_{1}\right) \leq v\left(x_{0}\right)+\frac{1}{2}$. Assume that we have construct a sequence $\left(x_{n}\right)$ such that for any $n$,

$$
x_{n+1} \in S\left(x_{n}\right), x_{n+1} \neq x_{n} \text { such that } f\left(x_{n+1}\right) \leq v\left(x_{n}\right)+\frac{1}{2^{n}} .
$$

Observe first that from Proposition 7, the sequence $\left(S\left(x_{n}\right)\right)$ is decreasing $\left(S\left(x_{m}\right) \subset S\left(x_{n}\right)\right.$ for $m \geq n$ ) so for any $m \geq n, p\left(x_{m}, x_{m}\right)=p\left(x_{n}, x_{n}\right)$. Moreover, applying the triangular inequality, we get for any $n$,

$$
\begin{aligned}
f\left(x_{n+2}\right)+p\left(x_{n}, x_{n+2}\right) & \leq f\left(x_{n+2}\right)+p\left(x_{n}, x_{n+1}\right)+p\left(x_{n+1}, x_{n+2}\right)-p\left(x_{n+1}, x_{n+1}\right) \\
& \leq f\left(x_{n+1}\right)+p\left(x_{n}, x_{n+1}\right) \\
& \leq f\left(x_{n}\right)+p\left(x_{n}, x_{n}\right)
\end{aligned}
$$

so by induction, one has

$$
f\left(x_{m}\right)+p\left(x_{n}, x_{m}\right) \leq f\left(x_{n}\right)+p\left(x_{n}, x_{n}\right)
$$

for any $n, m ; m \geq n$, or equivalently

$$
0 \leq p\left(x_{n}, x_{m}\right)-p\left(x_{n}, x_{n}\right) \leq f\left(x_{n}\right)-f\left(x_{m}\right)
$$

Observe that (9) implies that $\left(f\left(x_{n}\right)\right)$ is a decreasing sequence of real numbers and as it is bounded from below, the sequence $\left(f\left(x_{n}\right)\right)$ is convergent. Therefore,

$$
\lim _{n, m \rightarrow \infty}\left(p\left(x_{n}, x_{m}\right)-p\left(x_{n}, x_{n}\right)\right)=0 .
$$

Thus $\lim _{n, m \rightarrow \infty} d_{p}\left(x_{n}, x_{m}\right)=2 \lim _{n, m \rightarrow \infty}\left(p\left(x_{n}, x_{m}\right)-p\left(x_{n}, x_{n}\right)\right)=0$ hence $\left(x_{n}\right)$ is a Cauchy sequence in $\left(X, d_{p}\right)$ which is complete (by Lemma 5). There exists then $\bar{x} \in X$ such that $\lim _{n \rightarrow \infty} d_{p}\left(x_{n}, \bar{x}\right)=0$ so from (3),

$$
p(\bar{x}, \bar{x})=\lim _{n \rightarrow \infty} p\left(\bar{x}, x_{n}\right)=\lim _{n, m \rightarrow \infty} p\left(x_{n}, x_{m}\right) .
$$

On the other hand, since $S\left(x_{n+m}\right) \subset S\left(x_{n}\right)$ for any $n, m$ so that the sequence $\left(x_{n+m}\right)_{m}$ is in $S\left(x_{n}\right)$ and satisfies $\lim _{m \rightarrow \infty} d_{p}\left(x_{n+m}, \bar{x}\right)=0$ for any $n$ fixed thus $\bar{x} \in S\left(x_{n}\right)$ for all $n\left(S\left(x_{n}\right)\right.$ is closed). Particularly, $\bar{x} \in S\left(x_{0}\right)$ that is $\bar{x}$ satisfies $p(\bar{x}, \bar{x})=p\left(x_{0}, x_{0}\right)$ and $f(\bar{x})+p\left(\bar{x}, x_{0}\right) \leq f\left(x_{0}\right)+p\left(x_{0}, x_{0}\right)$.

Let us prove now that $\bar{x}$ is a $p$-point of $f$. Consider a point $y$ in $S(\bar{x})$ with $y \neq \bar{x}$. Then $y \in S\left(x_{n}\right) \subset S\left(x_{n-1}\right)$ for any $n \geq 1$ and using (7), we get

$$
f(y) \leq f(y)+p\left(x_{n}, y\right)-p\left(x_{n}, x_{n}\right) \leq f\left(x_{n}\right) \leq v\left(x_{n-1}\right)+\frac{1}{2^{n}} \leq f(y)+\frac{1}{2^{n}}
$$


thus

$$
0 \leq p\left(x_{n}, y\right)-p\left(x_{n}, x_{n}\right) \leq \frac{1}{2^{n}} .
$$

We conclude that $\lim _{n \rightarrow \infty} p\left(x_{n}, y\right)=p(\bar{x}, \bar{x})$ since $\lim _{n \rightarrow \infty} p\left(x_{n}, x_{n}\right)=p(\bar{x}, \bar{x})$. As

$$
p(\bar{x}, y)+p\left(x_{n}, x_{n}\right)-p\left(\bar{x}, x_{n}\right) \leq p\left(x_{n}, y\right) \leq p(\bar{x}, y)+p\left(x_{n}, \bar{x}\right)-p(\bar{x}, \bar{x})
$$

$\lim _{n \rightarrow \infty} p\left(x_{n}, y\right)=p(\bar{x}, y)$. Thus $p(\bar{x}, y)=p(\bar{x}, \bar{x})=p(y, y)(y \in S(\bar{x}))$ and hence $y=\bar{x}$ from (i) of Definition 2. This leads that $\bar{x}$ is a $p$-point.

The converse of the above theorem is as the following:

Theorem 9. A partial metric space $(X, p)$ is complete iffor every proper, lower semicontinuous on $(X, p)$ and bounded from below function $f: X \rightarrow \mathbb{R} \cup\{+\infty\}$ and for every $\varepsilon>0$ there exists $x_{\varepsilon} \in X$ such that

$$
f\left(x_{\varepsilon}\right) \leq \inf _{x \in X} f(x)+\varepsilon
$$

and

$$
f\left(x_{\varepsilon}\right)+\varepsilon p\left(x_{\varepsilon}, x_{\varepsilon}\right) \leq f(x)+\varepsilon p\left(x, x_{\varepsilon}\right) \quad \forall x \in X .
$$

Proof. Consider $\left(x_{n}\right)$ a Cauchy sequence on $(X, p)$ and define a function $f: X \rightarrow \mathbb{R} \cup\{+\infty\}$ such as $f(x):=\lim _{n \rightarrow \infty} p\left(x_{n}, x\right)-p(x, x)$ for any $x \in X$. Then $f$ is proper, nonnegative and l.s.c. on $(X, p)$. Indeed, let $y \in X$ and take a sequence $\left(y_{m}\right)$ such that $\lim _{m \rightarrow \infty} p\left(y_{m}, y\right)=p(y, y)$. Since for any $n$ and $m$,

$$
p\left(x_{n}, y\right)-p(y, y) \leq p\left(x_{n}, y_{m}\right)+p\left(y_{m}, y\right)-p\left(y_{m}, y_{m}\right)-p(y, y)
$$

we obtain for $n \rightarrow \infty$,

$$
f(y) \leq f\left(y_{m}\right)+p\left(y_{m}, y\right)-p(y, y)
$$

and thus

$$
f(y) \leq \liminf _{m \rightarrow \infty} f\left(y_{m}\right) .
$$

Moreover, since $\left(x_{n}\right)$ is a Cauchy sequence thus $\lim _{m \rightarrow \infty} f\left(x_{m}\right)=0$ by using the fact that

$$
\lim _{n, m \rightarrow \infty} p\left(x_{n}, x_{m}\right)=\lim _{m \rightarrow \infty} p\left(x_{m}, x_{m}\right) \in \mathbb{R}
$$

Hence $\inf _{x \in X} f(x)=0$.

Given $\varepsilon \in(0,1)$ fixed so by assumption there exists $\bar{x} \in X$ such as $f(\bar{x}) \leq \varepsilon$ and

$$
f(\bar{x})+\varepsilon p(\bar{x}, \bar{x}) \leq f(x)+\varepsilon p(x, \bar{x}) \quad \forall x \in X
$$

thus

$$
f(\bar{x}) \leq f\left(x_{n}\right)+\varepsilon\left(p\left(x_{n}, \bar{x}\right)-p(\bar{x}, \bar{x})\right) .
$$


Taking the limit for $n \rightarrow \infty$, we get that $f(\bar{x}) \leq \varepsilon f(\bar{x})$ and so $f(\bar{x})=0$ that is $\lim _{n \rightarrow \infty} p\left(x_{n}, \bar{x}\right)=$ $p(\bar{x}, \bar{x})$. We conclude that $\left(x_{n}\right)$ converges to $\bar{x}$ and $(X, p)$ is complete.

\section{Fixed point of generalized contraction set-valued mappings}

The existence of fixed-points for various set-valued contractive mappings had been studied by many authors under different conditions. In 1969, Nadler [32] extended the famous Banach contraction principle from single-valued mapping to set-valued mappings. The fixed point theory of set-valued contractions initiated by Nadler was developed in different directions by many authors, in particular, by Reich [41, 1972], Mizoguchi-Takahashi [31, 1989], Takahashi [46, 1991], Azé-Penot [9, 2006], Feng-Liu [21, 2006], Benahmed-Azé [11, 2010].

Recently, Aydi et al. [4, 2013] introduced the concept of a partial Hausdorff metric and extended the Nadler's fixed point theorem on partial metric spaces using the partial Hausdorff metric.

Our purpose now is to establish a fixed point theorem on partial metric spaces using our previous version of the Ekeland's variational principle. We obtain then a new generalization of Aydi's result [4] and Benahmed's result [11].

Let us recall the partial Hausdorff metric on a partial metric space $(X, d)$. Given $A, B$ two nonempty subsets of $X$, the partial excess of $A$ on $B$ is defined as

$$
e_{p}(A, B):=\sup (p(x, B), x \in A)
$$

where $p(x, B):=\inf (p(x, y), y \in B)$. It is proved in [4], that $e_{p}$ satisfies the following properties:

$$
\begin{aligned}
& e_{p}(A, A)=\sup _{a \in A} p(a, a), \\
& e_{p}(A, A) \leq e_{p}(A, B), \\
& e_{p}(A, B)=0 \Longrightarrow A \subseteq B, \\
& e_{p}(A, B) \leq e_{p}(A, C)+e_{p}(C, B)-\inf _{c \in C} p(c, c)
\end{aligned}
$$

for any $A, B, C \in C B^{p}(X)$ where $C B^{p}(X)$ is the family of all nonempty, closed and bounded subsets of the partial metric space $(X, p)$. Note that closedness is with respect to the partial metric $p: A$ is closed on $\left(X, \tau_{p}\right)$ if $\bar{A}=A$, and boundedness is given as follows: $A$ is a bounded subset in $(X, p)$ if there exists a ball $B_{p}\left(x_{0}, r\right)$ such that $A \subset B_{p}\left(x_{0}, r\right)$ that is $p\left(x_{0}, a\right)<p(a, a)+r$ for any $a \in A$.

Consider now the partial Hausdorff metric of $A$ and $B$ defined in [4] which is given by

$$
h_{p}(A, B):=\max \left(e_{p}(A, B), e_{p}(B, A)\right)
$$

for any $A, B \in C B^{p}(X)$. 
The next result is the generalized Banach contraction theorem given recently by Aydi et al. [4]:

Theorem 10. Let $(X, p)$ be a complete partial metric space and let $k \in[0,1)$. If $T: X \rightarrow C B^{p}(X)$ is a set-valued mapping such that for all $x, y \in X$, we have

$$
h_{p}(T x, T y) \leq k p(x, y)
$$

then $T$ has a fixed point that is there exists $x \in X$ such that $x \in T x$.

Let us introduce the following generalized contraction property of set valued mapping on partial metric spaces: given a set-valued mapping $T: X \rightrightarrows X$ with nonempty values, we will say that $T$ is a $p$-generalized contraction if there exist $\theta, \kappa \in(0,1)$ so that for any $x \in X$ such that $p(x, T x)>p(x, x)$ there exists $y \in T x$ with $y \neq x$ and $p(y, y)=p(x, x)$ which satisfies

$$
\left\{\begin{array}{l}
(i) \kappa p(x, y)+(1-\kappa) p(x, x)<p(x, T x) \leq p(x, y) \\
\text { (ii) } p(y, T y) \leq \theta p(x, y)+(1-\theta) p(x, x) .
\end{array}\right.
$$

When we consider the partial metric as a metric in (10), we recover as a particular case the notion of generalized set-valued contraction mappings introduced by Benahmed-Azé [11].

Let us denote by $\mathscr{F}_{T}:=\{x \in X: x \in T x\}$ the fixed points set of $T$ and observe that when each value of $T$ is closed with respect to the topology $\tau_{p}$ one has

$$
\mathscr{F}_{T}:=\{x \in X: p(x, T x)=p(x, x)\}
$$

since we have the equivalence (see [4, Remark 2.1])

$$
a \in \bar{A} \Longleftrightarrow p(a, A)=p(a, a)
$$

for any nonempty set $A \subset X$.

Our first statement is to show that every set-valued mapping $T: X \rightrightarrows X$ which is a $p$-generalized contraction satisfies an approximate fixed point property (see [16]) that is

$$
\inf _{x \in X}(p(x, T x)-p(x, x))=0
$$

or equivalently, for any $\varepsilon>0$ there exists $x_{\varepsilon} \in X$ such that

$$
p\left(x_{\varepsilon}, T x_{\varepsilon}\right)<p\left(x_{\varepsilon}, x_{\varepsilon}\right)+\varepsilon .
$$

Remark that $p(x, x) \leq p(x, T x)$ since $p(x, x) \leq p(x, y)$ for any $x, y \in X$. 
Proposition 11. Let $(X, p)$ be a partial metric space and $T: X \rightrightarrows X$ a $p$-generalized contraction set valued mapping with closed values and such that $\theta<\kappa$. Then $\inf _{x \in X}(p(x, T x)-p(x, x))=0$.

Proof. Given $x_{0} \in X$ such that $p\left(x_{0}, T x_{0}\right)>p\left(x_{0}, x_{0}\right)$. From (10), we can pick $x_{1} \in T\left(x_{0}\right), x_{1} \neq x_{0}$ and $p\left(x_{1}, x_{1}\right)=p\left(x_{0}, x_{0}\right)$ so that

$$
\left\{\begin{array}{l}
\kappa p\left(x_{0}, x_{1}\right)+(1-\kappa) p\left(x_{0}, x_{0}\right)<p\left(x_{0}, T x_{0}\right) \\
p\left(x_{1}, T x_{1}\right) \leq \theta p\left(x_{0}, x_{1}\right)+(1-\theta) p\left(x_{0}, x_{0}\right) .
\end{array}\right.
$$

Note that $p\left(x_{1}, x_{1}\right) \leq p\left(x_{1}, T x_{1}\right)$. If $p\left(x_{1}, T x_{1}\right)=p\left(x_{1}, x_{1}\right)$, we get the conclusion of the lemma. Assume now that $p\left(x_{1}, T\left(x_{1}\right)\right)>p\left(x_{1}, x_{1}\right)$ and suppose we have constructed a finite sequence $\left(x_{i}\right)_{i=0, . ., n}$ such that for all $i \in\{0, \ldots, n\}, p\left(x_{i}, T x_{i}\right)>p\left(x_{i}, x_{i}\right)$ and for $i \in\{0, \ldots, n-1\}, x_{i+1} \in T x_{i}$ $\left(x_{i+1} \neq x_{i}, p\left(x_{i+1}, x_{i+1}\right)=p\left(x_{i}, x_{i}\right)\right)$ with

$$
\left\{\begin{array}{c}
\kappa p\left(x_{i}, x_{i+1}\right)+(1-\kappa) p\left(x_{i}, x_{i}\right)<p\left(x_{i}, T x_{i}\right) \\
p\left(x_{i+1}, T x_{i+1}\right) \leq \theta p\left(x_{i}, x_{i+1}\right)+(1-\theta) p\left(x_{i}, x_{i}\right) .
\end{array}\right.
$$

Applying assumption (10), one can find $x_{n+1} \in T x_{n}$ such that $x_{n+1} \neq x_{n}, p\left(x_{n+1}, x_{n+1}\right)=$ $p\left(x_{n}, x_{n}\right)$ and

$$
\left\{\begin{array}{c}
\kappa p\left(x_{n}, x_{n+1}\right)+(1-\kappa) p\left(x_{n}, x_{n}\right)<p\left(x_{n}, T x_{n}\right) \\
p\left(x_{n+1}, T x_{n+1}\right) \leq \theta p\left(x_{n}, x_{n+1}\right)+(1-\theta) p\left(x_{n}, x_{n}\right) .
\end{array}\right.
$$

If $p\left(x_{n+1}, T x_{n+1}\right)=p\left(x_{n+1}, x_{n+1}\right)$ then $\inf _{x \in X}(d(x, T x)-p(x, x))=0$.

Suppose $p\left(x_{n+1}, T x_{n+1}\right)>p\left(x_{n+1}, x_{n+1}\right)$. By induction, either the process stops if there exists $k \in \mathbb{N}^{*}$ such that $p\left(x_{k}, T x_{k}\right)=p\left(x_{k}, x_{k}\right)$ or we construct a sequence $\left(x_{n}\right)$ satisfying $p\left(x_{n}\right.$, $\left.T x_{n}\right)>p\left(x_{n}, x_{n}\right)$ and (14) for all $n$. Let us set $\delta_{n}:=p\left(x_{n}, T x_{n}\right)-p\left(x_{n}, x_{n}\right)$ and $\mu_{n}:=p\left(x_{n}, x_{n+1}\right)-$ $p\left(x_{n}, x_{n}\right)$. Observe that $0 \leq \delta_{n} \leq \mu_{n}$ since $x_{n+1} \in T x_{n}$. Moreover, as $\theta<\kappa$, we get from (13) and (14) and the fact that $p\left(x_{n}, x_{n}\right)=p\left(x_{n+1}, x_{n+1}\right)$,

$$
\begin{aligned}
\delta_{n+1} & \leq \theta\left(p\left(x_{n}, x_{n+1}\right)-p\left(x_{n}, x_{n}\right)\right) \\
& \leq \kappa\left(p\left(x_{n}, x_{n+1}\right)-p\left(x_{n}, x_{n}\right)\right) \leq \delta_{n}
\end{aligned}
$$

and

$$
\begin{aligned}
\mu_{n} & <\kappa^{-1}\left(p\left(x_{n}, T x_{n}\right)-p\left(x_{n}, x_{n}\right)\right) \\
& \leq \theta \kappa^{-1}\left(p\left(x_{n}, x_{n-1}\right)-p\left(x_{n-1}, x_{n-1}\right)\right) \leq \mu_{n-1}
\end{aligned}
$$

so that $\left(\delta_{n}\right)$ and $\left(\mu_{n}\right)$ are nonnegative decreasing sequences in $\mathbb{R}$. Hence $\left(\delta_{n}\right)$ and $\left(\mu_{n}\right)$ converge to some real number $\bar{\delta}$ and $\bar{\mu}$ respectively which satisfy $0 \leq \bar{\delta} \leq \bar{\mu}$. If $\bar{\delta}>0$ it yields, from the following inequalities,

$$
\delta_{n+1} \leq \theta\left(p\left(x_{n}, x_{n+1}\right)-p\left(x_{n}, x_{n}\right)\right) \leq \frac{\theta}{\kappa} \kappa\left(p\left(x_{n}, x_{n+1}\right)-p\left(x_{n}, x_{n}\right)\right)
$$




$$
\leq c\left(p\left(x_{n}, T x_{n}\right)-p\left(x_{n}, x_{n}\right)\right) \leq c \delta_{n}
$$

the contradiction $\bar{\delta} \leq c \bar{\delta}<\bar{\delta}$ since $c=\frac{\theta}{\kappa}<1$. We conclude that $\lim _{n \rightarrow \infty}\left(p\left(x_{n}, T x_{n}\right)-p\left(x_{n}, x_{n}\right)\right)=0$ and thus the announced result follows.

Let us remark that from (15), $\lim _{n \rightarrow \infty}\left(p\left(x_{n}, x_{n+1}\right)-p\left(x_{n}, x_{n}\right)\right)=0$.

The next result is devoted to the existence of fixed points for generalized contraction on partial space.

Theorem 12. Given a complete partial metric space $(X, p)$ and let $T: X \rightrightarrows X$ be a set-valued map with closed and nonempty values. Assume that:

(i) $T$ is a $p$-generalized contraction with $0<\theta<\kappa<1$,

(ii) the function $x \rightarrow p(x, T x)-p(x, x)$ is lower semicontinuous.

Then $\mathscr{F}_{T} \neq \varnothing$ and for any $x \in X$ such that $p(x, T x)>p(x, x)$,

$$
(\kappa-\theta) p\left(x, \mathscr{F}_{T}\right) \leq p(x, T x)-(1-(\kappa-\theta)) p(x, x) .
$$

Proof. Define $f: X \rightarrow \mathbb{R}$ as $f(x):=(\kappa-\theta)^{-1}(p(x, T x)-p(x, x))$ which is nonnegative and lower semicontinuous (by assumption (ii)). Hence by Theorem $8, f$ admits a $p$-point denoted by $\bar{x}$. Otherwise, for any $x \in X$ such that $p(x, T x)>p(x, x)$, there exists from assumption (i), $y \in T x$ (with $y \neq x$ and $p(y, y)=p(x, x)$ ) such that

$$
\left\{\begin{array}{l}
\kappa p(x, y)+(1-\kappa) p(x, x) \leq p(x, T x) \\
p(y, T y) \leq \theta p(x, y)+(1-\theta) p(x, x) .
\end{array}\right.
$$

Hence

$$
p(y, T y)+(\kappa-\theta) p(x, y) \leq p(x, T x)+(\kappa-\theta) p(x, x)
$$

or equivalently,

$$
f(y)+p(x, y) \leq f(x)+p(x, x) .
$$

We conclude that any $x$ such that $p(x, T x)>p(x, x)$ isn't a $p$-point of $f$ thus $d(\bar{x}, T \bar{x}) \leq$ $p(\bar{x}, \bar{x})$. Because $d(\bar{x}, \bar{x}) \leq p(\bar{x}, T \bar{x})$, we obtain that $p(\bar{x}, T \bar{x})=p(\bar{x}, \bar{x})$ and from $(12,11), \mathscr{F}_{T} \neq$ $\varnothing$. In the same way, any $p$-point $y$ of $f$ in $S(x)$ (which exists by Theorem 8) with $p(x, T(x))>p(x, x)$ is such as $y \in \mathscr{F}_{T}$ and satisfies

$$
p(x, y) \leq f(y)+p(x, y) \leq f(x)+p(x, x)
$$


thus

$$
(\kappa-\theta) p\left(x, \mathscr{F}_{T}\right) \leq p(x, T x)-(1-(\kappa-\theta)) p(x, x)
$$

and the theorem is proved.

Note that the assumption (ii) is satisfied whenever one has

$$
p(x, y) \rightarrow p(x, x) \Longrightarrow e_{p}(T y, T x) \rightarrow 0
$$

since

$$
\begin{aligned}
p(x, T x)-p(x, x) & \leq p(x, y)+p(y, T x)-p(y, y)-p(x, x) \\
& \leq[p(x, y)-p(x, x)]+[p(y, T y)-p(y, y)]+e_{p}(T y, T x)
\end{aligned}
$$

by using the fact that

$$
p(y, T x) \leq p(y, T y)+e_{p}(T y, T x)-\inf _{z \in T y} p(z, z) \leq p(y, T y)+e_{p}(T y, T x) .
$$

Remark 13. Notice that the previous theorems can be generalized for set-valued mappings $T$ satisfying the following $p$-generalized contraction condition: for any $x \in X$ such that $p_{x}:=$ $p(x, T x)-p(x, x)>0$ there exists $y \in T x$ with $y \neq x$ and $p(y, y)=p(x, x)$ so that

$$
\left\{\begin{array}{l}
(i) \kappa\left(p_{x}\right) p(x, y)+\left(1-\kappa\left(p_{x}\right)\right) p(x, x)<p(x, T x) \leq p(x, y) \\
\text { (ii) } p(y, T y) \leq \theta(p(x, y)) p(x, y)+(1-\theta(p(x, y))) p(x, x)
\end{array}\right.
$$

where $\kappa(\cdot):(0,+\infty) \rightarrow[\bar{\kappa}, 1]$ with $\bar{\kappa} \in(0,1]$ is a nonincreasing function and $\theta(\cdot):(0,+\infty) \rightarrow[0,1)$ such as $\theta(\cdot)<\kappa(\cdot)$ and $\lim \sup \theta(t) / \kappa(t)<1$ for any $s>0$.

$$
t \downarrow s
$$

\section{References}

[1] J.-P. Aubin, Mathematical Methods of Game and Economic Theory, North-Holland Publishing, Amsterdam, New York, Oxford, 1979.

[2] J.-P. Aubin and I. Ekeland, Applied Nonlinear Analysis Wiley, New York, Chichester, Brisbane, Toronto, Singapore, 1984.

[3] J.-P. Aubin and H. Frankowska, Set-Valued Analysis, Birkhäuser, Boston, Basel, Berlin, 1990.

[4] H. Aydi, M. Abbas and C. Vetro, Partial Hausdorff metric and Nadler's fixed point theorem on partial metric spaces, Topology and its Applications, 2012, http://dx . doi .org/10.1016/j . topol .2012.06.012.

[5] D. Azé, J.-N. Corvellec and R. E. Lucchetti, Variational pairs and applications to stability in nonsmooth analysis, Nonlinear Anal., 49 (2002), 643-670.

[6] D. Azé and J.-N. Corvellec, Characterizations of error bounds for lower semicontinuous functions on metric spaces, ESAIM Control Optim. Calc. Var., 10 (2004), 409-425.

[7] D. Azé and J.-N. Corvellec, A variational method in fixed point results with inwardness conditions, Proc. Amer. Math. Soc., 134 (2006), 3577-3583.

[8] D. Azé and J.-N. Corvellec, Variational methods in classical open mapping theorems, J. Convex Anal., 13 (2006). 
[9] D. Azé and J.-P. Penot, On the dependence of fixed point sets of pseudocontractive multifunctions. Application to differential inclusions, Nonlinear Dyn. Syst. Theory, 6(2006), 31-47.

[10] S. Banach, Sur les opérations dans les ensembles abstraits et leur application aux équations intégrales, Fundam. Math., 3(1922), 133-181.

[11] S. Benahmed and D. Azé, On fixed points of generalized set-valued contractions, Bulletin of the Australian Mathematical Society, 2010.

[12] J. M. Borwein and D. Preiss, A smooth variational principle with applications to subdifferentiability and to differentiability of convex functions, Trans. Am. Math. Soc., 303(1987), 517-527.

[13] J. M. Borwein and Q. J. Zhu, Techniques of Variational Analysis, Springer, Berlin, Heidelberg, New York, 2005.

[14] Lj.B. Ćirić, A Generalization of Banach's contraction principle, Proc. Amer. Math. Soc., 45(1974), $267-273$.

[15] Lj. B. Ćirić, B. Samet, H. Aydi and C. Vetro, Common fixed points of generalized contractions on partial metric spaces and an application, Appl. Math. Comput., 218(2011), 2398-2406.

[16] W. S. Du, On approximate coincidence point properties and their applications to fixed point theory, Journal of Applied Mathematics, 2012, doi :10.1155/2012/302830.

[17] I. Ekeland, On the variational principle, J. Math. Anal. Appl., 47(1974), 324-353.

[18] I. Ekeland, On convex minimization problems, Bull. Am. Math. Soc., 1(1979), 445-474.

[19] I. Ekeland, Nonconvex minimization problems, Bull. Amer. Math. Soc., 1(1979), 443-474.

[20] F. Facchinei and J.-S. Pang, Finite Dimensional Variational Inequalities and Complementarity Problems, I and II. Springer, New York, Berlin, Heidelberg, 2003.

[21] Y. Feng and S. Liu, Fixed point theorems for multi-valued contractive mappings and multi-valued Caristi type mappings, J. Math. Anal. Appl., 317(2006), 103-112.

[22] D. G. de Figueiredo, Lectures on the Ekeland variational principle with applications and detours, Tata Institute of Fundamental Research Lectures on Mathematics and Physics, 81, Springer-Verlag, Berlin, 1989.

[23] A. Göfert, Chr. Tammer, H. Riahi and C. Zălinescu, Variational Methods in Partially Ordered Spaces. Springer, New York, Berlin, Heidelberg, 2003.

[24] A. Hamel, Remarks to an equivalent formulation of Ekeland's variational principle, Optimization 31(1994), 233-238.

[25] R. Kannan, Some results on fixed points, Bull. Cal. Math. Soc., 60(1968), 71-76.

[26] E. Karapınar, Generalizations of Caristi Kirk's theorem on partial metric spaces, Fixed Point Theory Appl., 4 (2011), DOI : $10.1186 / 1687-1812-2011-4$.

[27] I. Meghea, Ekeland Variational Principle with Generalizations and Variants. Old City Publishing, Philadelphia, 2009.

[28] S. G. Matthews, Partial metric topology. Research Report 212. Dept. of Computer Science. University of Warwick, 1992.

[29] S. G. Matthews, Partial metric topology, in: Proc. 8th Summer Conference on General Topology and Applications, in: Ann. New York Acad. Sci., 728(1994), 183-197.

[30] S. G. Matthews, An extensional treatment of lazy data flow deadlock, Theor. Comput. Sci., 151(1995), 195-205.

[31] N. Mizoguchi and W. Takahashi, Fixed point theorems for multivalued mappings on complete metric spaces, J. Math. Anal Appl., 141(1989), 177-188.

[32] S. B. Jr. Nadler, Multivalued contraction mappings, Pacific J. Math., 30(1969), 475-488.

[33] W. Oettli and M. Théra, Equivalents of Ekeland's principle, Bull. Aust. Math. Soc., 48(1993), 385-392.

[34] J.-P. Penot, A short constructive proof of Caristi's fixed point theorem, Publ. Math. Univ. Pau, (1976), 1-3.

[35] J.-P. Penot, The drop theorem, the petal theorem and Ekeland's variational principle, Nonlinear Anal., 10(1986), 813-822.

[36] S. Oltra and O. Valero, Banach's fixed point theorem for partial metric spaces, Rend. Istit. Mat. Univ. Trieste., 36(2004), 17-26.

[37] D. Paesano and P. Vetro, Suzuki's type characterizations of completeness for partial metric spaces and fixed points for partially ordered metric spaces, Topology Appl., 159(2012), 911-920.

[38] S. Romaguera, Fixed point theorems for generalized contractions on partial metric spaces, Topology Appl., 159(2012), 194-199. 
[39] I. A. Rus, Fixed point theory in partial metric spaces, Anal. Univ. de Vest, Timisoara, Seria MatematicăInformatică, 46 (2008), 141-160.

[40] J. E. Stoy, Denotational Semantics: The Scott-Strachey Approach to Programming Language Theory, MIT Press Cambridge, 1981.

[41] S. Reich, Kannan's fixed point theorem, Boll. Un. Mat. Ital., 4(1971), 1-11.

[42] M. Squassina, On Ekeland's variational principle, J. Fixed Point Theor. Appl., 10 (2011), 191-195.

[43] T. Suzuki, Generalized Caristi's fixed point theorems by Bae and others, J. Math. Anal. Appl., 302 (2005), $502-$ 508.

[44] T. Suzuki, The strong Ekeland variational principle, J. Math. Anal. Appl., 320(2006), 787-794.

[45] T. Suzuki and W. Takahashi, Fixed point theorems and characterizations of metric completeness, Top. Meth. Nonlinear Anal., 8 (1996), 371-382.

[46] W. Takahashi, Existence theorems generalizing fixed point theorems for multivalued mappings. In: Baillon, J.-B., Théra, M. (eds.) Fixed Point Theory and Applications. Pitman Research Notes in Mathematics, 252 (1991), 397-406. Longman, Harlow.

[47] W. Takahashi, Nonlinear Functional Analysis. Yokohama Publishers, Yokohama, 2000.

[48] Chr. Tammer, A generalization of Ekeland's variational principle, Optimization, 25 (1992), 129-141.

[49] O. Valero, On Banach fixed point theorems for partial metric spaces, Appl. General Topology, 6 (2005), 229240.

[50] L. Yongsin and S. Shuzhong, A generalization of Ekeland's $\varepsilon$-variational principle and its Borwein-Preiss variant, J. Math. Anal. Appl., 246 (2000), 308-319.

[51] C.-K. Zhong, On Ekeland's variational principle and a minimax theorem, J. Math. Anal. Appl., 205 (1997), 239-250.

[52] C.-K. Zhong, A generalization of Ekeland's variational principle and application to the study of the relation between the P.S. condition and coercivity, Nonlinear Anal. Theor. Meth. Appl., 29 (1997), 1421-1431.

Department of Mathematics, Sciences Exactes Faculty, Biskra University, Algeria.

E-mail: djedidim@yahoo.fr

Camille Jordan Institute, Lyon, French and Laboratory of Operator Theory, Eloued University, Algeria.

E-mail: nachikh@gmail.com

Laboratory of Mathematics and its Applications, University of Oran, BP 1524 El M'naouer, Algeria

E-mail: amansour@math.univ-lyon1.fr 